\title{
Miller Fisher Variant of Guillain-Barré Syndrome: A Case Report \& Clinical Review
}

\author{
Partha Pratim Das 1 , Sarmistha Biswash', Md. Enamul Karim², Nigar Sultana Ahmed ${ }^{3}$, Badrul Islam ${ }^{4}$, Shekhar \\ Kumar Mandal', Abu Hena Md. Raihanuzzaman Sarkar ${ }^{5}$ \\ ${ }^{1}$ Assistant Professor, ${ }^{2}$ Professor, ${ }^{3}$ Assistant Registrar, ${ }^{5}$ Medical Officer, Department of Medicine, Dhaka Medical College, ${ }^{4}$ PHD Student, \\ Erasmus MC, Dhaka
}

\begin{abstract}
Miller Fisher syndrome (MFS) is an uncommon variant of Guillain-Barré syndrome (GBS). It is characterized by external ophthalmoplegia, ataxia, and areflexia. The incidence of MFS as a proportion of GBS was reported to be 1 to $5 \%$ in western countries and considerably higher in Eastern Asia i.e. 19\% in Taiwan and 25\% in Japan. Here we report the clinical features of a patient who initially presented with ataxia then subsequently developed limb weakness, areflexia and ophthalmoplegia. CSF study and NCV study supported our clinical diagnosis. Patient was managed conservatively. No intravenous immunoglobulin was given but the patient gradually improved in symptoms including power, ataxia, ophthalmoparesis. After one month the patient was discharged from hospital with complete recovery.
\end{abstract}

Keywords: Abnormal reflex, areflexia, ataxia, Guillain-Barré syndrome, ophthalmoplegia, Nerve Conduction Velocity (NCV).

[BSMMU J 2012; 5(1):69-71]

\section{Introduction:}

Miller Fisher Variant of Guillain-Barré Syndrome/ Miller Fisher syndrome (MFS) by itself is a rare entity. In 1932, Collier first described the triad of ataxia, areflexia and ophthalmoplegia as a variant of the Guillain-Barre syndrome. In 1956, Charles Miller Fisher, a Canadian stroke specialist, described 3 patients with acute external ophthalmoplegia, sluggish pupillary reflexes, ataxia and areflexia. ${ }^{1}$ All 3 patients recovered spontaneously. Because some GBS patients had ophthalmoplegia and other similarities, Fisher concluded that these patients had suffered a disorder akin to GBS. The syndrome was later called Miller Fisher syndrome. ${ }^{1}$ It is an uncommon clinical variant in Bangladesh. A case of Miller Fisher variant with GBS overlap having ataxia, areflexia, ophthalmoplegia and weakness is reported here.

\section{Case Report:}

A 39-year-old married man admitted to Dhaka Medical College Hospital Medicine Unit-II on 26 ${ }^{\text {th }}$ June 2011 with unsteadiness of gait for 2 days. Initially he noticed imbalance and tendency to fall on either side during walking. He had tingling sensation of all four limbs from the beginning. On $2^{\text {nd }}$ day of hospital admission he noticed diplopia which occurred when looking to right or left, difficulty in vision along with weakness in both

Address for Correspondence: Dr. Partha Pratim Das, Assistant Professor, Department of Medicine, Dhaka Medical College. E-mail:dmck53babun@yahoo.com upper \& lower limbs. Gradually the patient became bedridden at 4 th day. There were no complaints of unconsciousness, convulsion, speech problems, hearing defects, vertigo, tinnitus, dysphagia or hoarseness of voice. Bladder and bowel functions were normal. He had no history of fever, loose motion and upper respiratory tract infection in last one month. He had no recent history of vaccination, any surgery, drug abuse, alcohol addiction and extramarital sexual exposure. His family history was unremarkable.

On examination, the patient was conscious and cooperative. Pallor, cyanosis, jaundice, clubbing and lymphadenopathies were absent. His blood pressure was $125 / 70 \mathrm{mmHg}$ with no orthostatic fall. His pulse was 84/ minute and regular. Respiratory rate was 20/minute.

On the first day of admission, on neurological examination, his higher psychic function including speech and language was normal. Cranial nerve examination revealed decreased movements present on lateral, medial and upward gaze. Nystagmus was absent. Optic nerve including fundus was normal. Rests of the cranial nerves were normal to examination. On motor system examination, tone of the muscles of the lower limbs was reduced. Power of lower limbs was $4 / 5$ in both sides on the first day of admission. Power of upper limbs was normal initially. There was no wasting of muscles or any involuntary movement. Deep tendon jerks were absent in all four limbs. Planter was bilaterally flexor. Abdominal reflex was preserved. On 
sensory examination, all modalities of sensations were preserved. Romberg's test was positive. At that time he had wide based ataxic gait with tendency to fall on either side. His finger-nose test was mildly impaired but heelshin test was grossly affected. There was no dysdiadochokinesia and rebound phenomenon. Examinations of other systems were normal. Tandem gait was impaired grossly.

On $2^{\text {nd }}$ day of hospital admission he developed bilateral ptosis. Eye movements were restricted in all direction. Pupillary response was bilaterally sluggish. Motor power of lower limbs deteriorated to $2 / 5$ and in upper limbs, motor power became $2 / 5$ on the lefts $3 / 5$ on the right side.

On admission, the complete blood counts (including platelet count), urea, creatinine and random sugar were all normal. His electrolyte was quite normal. In CSF study WBC count was $03 / \mathrm{cmm}$ and protein $180 \mathrm{mg} / \mathrm{dl}$. Nerve Conduction Velocity (NCV) study showed mixed demyelinating (both motor \& sensory) polyneuropathy. MRI scan of the brain was normal.

As the patient had ataxia, areflexia, mild ophthalmoparesis and weakness of lower limbs, clinically he was diagnosed to have Miller Fisher variant with GBS overlap. NCV study supported our clinical diagnosis. Patient was managed conservatively. No intravenous immunoglobulin was given but the patient gradually improved in symptoms including power, ataxia, ophthalmoparesis. After one month the patient was discharged from hospital with complete recovery.

\section{Discussion:}

Guillain-Barré syndrome (GBS), also known as acute idiopathic polyneuritis, is a type of neuromuscular paralysis that has several variants. ${ }^{1-4}$ Miller Fisher syndrome (MFS) is a rare variant of GBS, observed in only about $1 \%$ to $5 \%$ of all cases of GBS in Western countries. ${ }^{2,3}$ Miller Fisher syndrome occurs more in men than women with ratio of $2: 1.3$. The mean age of onset of MFS is 43.6 years, ${ }^{4}$ though onset has been documented in individuals between the ages of 13 and 78 years. ${ }^{3}$ In contrast, the incidence of MFS as a proportion of GBS was reported to be 1 to $5 \%$ in western countries and considerably higher in Eastern Asia i.e. $19 \%$ in Taiwan and $25 \%$ in Japan. 5,6

Berlit et al reviewed 223 cases of MFS. The first symptom was diplopia $(38.6 \%)$ or ataxia $(20.6 \%)$. Areflexia was present in $81.6 \%$ of cases. The cranial nerves other than III were involved in 127 cases (56.9\%): cranial nerves VII
(45.7\%), IX and X (39.9\%), and XII (13\%) were involved. In 53 cases, tetraparesis occurred. Elevated CSF protein was present in 134 patients (64.4\%). CSF findings were normal in 56 patients, and 18 patients had mild pleocytosis. The prognosis of MFS was good. Recovery occurred after a mean time period of 10.1 weeks. Residual symptoms were present in 74 cases (33.2\%), recurrence of MFS was reported in 7 patients, and 8 patients died. ${ }^{7}$

Fross et al reported 10 patients with typical MFS electrophysiological abnormalities characteristic of axonal neuropathy or neuronopathy with predominant sensory nerve changes in the limbs and motor damage in the cranial nerves. $^{8}$

On initial presentation, MFS may be mistaken for a brainstem stroke. However, the gradual onset of MFS in particular distinguishes this syndrome clinically from an acute stroke. The differential diagnosis of MFS includes Wernicke's encephalopathy and brainstem encephalitis, but these are associated with altered mental status. In addition, patients with Wernicke's encephalopathy usually have nystagmus, a feature not associated with MFS. Other entities to consider in the differential diagnosis of MFS are myasthenia gravis and other neuromuscular junction disorders. Appropriate laboratory and clinical electrophysiological testing with repetitive nerve stimulation helps to differentiate MFS from a neuromuscular junction disorder. ${ }^{9}$

The diagnosis of MFS is still descriptive depending on the presentation of the triad (ophthalmoplegia, ataxia and areflexia) of MFS. The combination of absent or reduced sensory responses on clinical electrophysiology testing and elevated protein with a normal white blood cell in CSF could lead to the diagnosis of MFS. ${ }^{10}$ Serial motor conduction velocities showed a marked reduction in the amplitudes of distal compound muscle action potential (CMAP), reaching low at 2 to 3 weeks, followed by a dramatic improvement in the 5 th week. Motor conduction velocities were in the normal range, with the distal motor latencies changing only slightly without the conduction block between the root and the distal nerves. These data might suggest an axonal neuropathy or a distal demyelinating conduction block. ${ }^{11}$ The diagnosis of MFS could be confirmed by positive anti-GQ1b antibody testing with a high level of sensitivity (well over $90 \%$ of patients with MFS have this antibody) and specificity. However, radiological imaging were unremarkable in most cases of MFS, lesions in the brainstem and spinocerebellar tracts had been reported in few patients. 
Different variants of MFS are present with a common tie of the GQ1b antibody. Some cases have only one or two symptoms out of the triad. The ataxia in MFS is due to peripheral mismatch between proprioceptive input from muscle spindles and kinesthetic information for joint receptors. ${ }^{1}$ Kusunoki et al found 5 patients with a variant form characterized by ataxia but no ophthalmoplegia in 149 patients who had the IgG anti-GQ1b antibody without profound weakness. These cases could fit with autoimmune ataxic neuropathy, having pathological lesions mainly in the dorsal root ganglion. GD1b antibody plays the key pathogenic role in autoimmune ataxic neuropathies. Because of the existence of cross-reactivity between GQ1b and GD1b, it is thought that the pathogenesis of this form is similar to that of MFS. ${ }^{12}$

Numerous case reports and series of patients with MFS treated with immunotherapy [generally plasma exchange, intravenous immunoglobulin G (IVIG) or a combination including one of these] have been reported. Analysis of the largest MFS case series failed to show any beneficial effects in the group who had received plasmapheresis when compared with the group who received no immunotherapy. ${ }^{6,13}$ Furthermore, in a recent randomised trial of patients with GBS, the addition of intravenous methylprednisolone with IVIG did not confer any additional effect on recovery from disability at 4 weeks, when compared with that of IVIG therapy alone. ${ }^{14}$

MFS is generally regarded as a self-limiting, benign condition. All of 28 untreated MFS patients in the largest published case series returned to normal activities 6 months after the neurological onset. The respective median (range) periods between neurological onset and the disappearance of ataxia and ophthalmoplegia were reported as 32 (8-271) and 88 (29-165) days. However, cases progressing to respiratory failure and requiring mechanical ventilation have also been described, particularly in children. Other serious complications reported include coma, ballism, cardiomyopathy from dysautonomia, lactic acidosis, and pain. ${ }^{15}$ Emergency physicians should aware of the severity of MFS, including that of bulbar palsy and/ or respiratory failure.

\section{Conclusion:}

High clinical suspicion is needed to diagnose Miller Fisher Variant of Guillain-Barré Syndrome because all symptoms may not appear at the same time. Though uncommon, respiratory failure is possible in MFS. In our case, ataxia was predominant initial signs. Eye signs were mild earlier but become profound later. Motor involvement was gradual and progressive and areflexia developed early. So, emergency physician should keep in mind that Ataxia may be earliest feature of MFS.

\section{References:}

1. Santra G, Datta AK. Miller Fisher syndrome-an uncommon clinical presentation. J Assoc Physicians India. 2008; 56:898900 .

2. Kozminski MP. Miller Fisher Variant of Guillain-Barré Syndrome: A Report of Case. J Am Osteopath Assoc. 2008; 108: $251-2$.

3. Mori M, Kuwabara S, Fukutake T, Yuki N, Hattori T. Clinical features and prognosis of Miller Fisher syndrome. Neurology. 2001; 56: 1104-6.

4. Berlit P, Rakicky J. The Miller Fisher syndrome. Review of literature. J Clin Neuroophthalmol. 1992; 12:57-63.

5. Mori M, Kuwabara S, Fukutake T, Yuki N, Hattori T. Clinical features and prognosis of Miller Fisher syndrome. Neurology. 2001;56(8):1104-6.

6. Overell JR, Willison HJ. Recent developments in Miller Fisher syndrome and related disorders. Curr Opin Neurol 2005, 18(5) :562-6.

7. Berlit P, Rakicky J. The Miller Fisher syndrome. Review of the literature. J Clin Neuroophthalmol. 1992; 12 :57-63.

8. Fross RD, Daube JR.Neuropathy in the Miller Fisher syndrome: clinical and electro physiologic findings. Neurology 1987; 37:1493-8.

9. Kuwabara S, Asahina M, Nakajima M, Mori M, Fukutake T, Hattori T, Yuki N. Special sensory ataxia in Miller Fisher syndrome detected by postural body sway analysis. Ann Neurol. 1999; 45 (4) : 533-536

10. Sever M, Aksay E, Gulec F. The diagnosis is made only with suspicion: Miller Fisher syndrome. Hong Kong j. emerg. Med. 2011. 18(6): 428-31

11. Uncini A, Lugaresi A. Fisher syndrome with tetraparesis and antibody to GQ $1 \mathrm{~b}$ : evidence for motor nerve terminal block. Muscle Nerve 1999; 22:640-4.

12. Kusunoki S, Chiba A, Kanazawa I. Anti-GQ1b IgG antibody is associated with ataxia as well as ophthalmoplegia. Muscle Nerve. 1999; 22:1071-4.

13. Mori M, Kuwabara S, Fukutake T, Hattori T. Plasmapheresis and Miller Fisher syndrome: analysis of 50 consecutive cases. J Neurol Neurosurg Psychiatry. 2002; 72(5):680.

14. van Koningsveld R, Schmitz PI, Meché FG, Visser LH, Meulstee J, van Doorn PA; Dutch GBS study group. Effect of methylprednisolone when added to standard treatment with intravenous immunoglobulin for Guillain-Barré syndrome: randomized trial. Lancet. 2004; 363(9404):192-6.

15. Lo YL. Clinical and immunological spectrum of the Miller Fisher syndrome. Muscle Nerve. 2007;36(5):615-27. 